\title{
Pacific
}

Journal of

Mathematics

\section{BIHARMONIC SURFACES OF CONSTANT MEAN CURVATURE}

\author{
ERIC LOUBeAu AND CEZAR ONICIUC
}

Volume $271 \quad$ No. 1 


\title{
BIHARMONIC SURFACES OF CONSTANT MEAN CURVATURE
}

\author{
ERIC LOUBEAU AND CEZAR ONICIUC
}

\begin{abstract}
We compute a Simons-type formula for the stress-energy tensor of biharmonic maps from surfaces. Specializing to Riemannian immersions, we prove several rigidity results for biharmonic CMC surfaces, putting in evidence the influence of the Gaussian curvature on pseudoumbilicity. Finally the condition of biharmonicity is shown to enable an extension of the classical Hopf theorem to CMC surfaces in any ambient Riemannian manifold.
\end{abstract}

\section{Introduction}

While harmonic maps between abstract Riemannian manifolds are a generalization of minimal submanifolds, their study on two-dimensional domains remained nonetheless very valuable and brought new light to both theories. When, for topological or geometrical reasons, harmonic maps are nonexistent or unsatisfactory, one can then measure the failure of harmonicity with the bienergy functional

$$
E_{2}(\phi)=\frac{1}{2} \int_{M}|\tau(\phi)|^{2} v_{g}
$$

where $M$ is compact, $\phi:(M, g) \rightarrow(N, h)$ is a smooth map and $\tau(\phi)=$ trace $\nabla d \phi$ is the tension field. Usual arguments (see [Jiang 1986]) show that critical points of $E_{2}$, called biharmonic maps, are solutions of

$$
\tau_{2}(\phi)=-\Delta \tau(\phi)-\operatorname{trace} R^{N}(d \phi(\cdot), \tau(\phi)) d \phi(\cdot)=0,
$$

and we will use the adjective proper to designate nonharmonic biharmonic maps.

Whilst the interconnections between harmonic maps and minimal surfaces are clear and well-established, in many cases, but not always, biharmonic Riemannian immersions have constant mean curvature (CMC). However, this link is not as clear as harmonicity and minimality, and the principal objective of this article is to explain how biharmonicity constrains CMC surfaces in an abstract ambient

C. Oniciuc was supported by a grant of the Romanian National Authority for Scientific Research, CNCS-UEFISCDI, project number PN-II-RU-TE-2011-3-0108.

MSC2010: 53C42, 53C43, 58E20.

Keywords: biharmonic maps, constant mean curvature, stress-energy tensor. 
manifold. This is particularly well-illustrated on compact biharmonic CMC surfaces whose Gaussian curvature has constant sign. They must be flat or pseudoumbilical if $K^{M}$ is nonnegative (Corollary 11); otherwise they have pseudoumbilical points (Theorem 12). The role of pseudoumbilical points in relaxing curvature constraints is further felt in the noncompact case, as their absence forces the CMC surface to be conformally flat (Theorem 12).

For complete surfaces, nonnegative Gaussian curvature and an upper bound on the sectional curvature of the ambient space will cause the surface to be flat or pseudoumbilical, but note that both can occur simultaneously (Proposition 18). When the ambient manifold is a three-dimensional space form, the surface must be umbilical (Corollary 14); consult [Montaldo and Oniciuc 2006] for the classification.

Our approach is to derive, in Proposition 3, a Simons-type formula for the biharmonic stress-energy tensor, valid for all smooth maps. As cumbersome as this equation is in the general case, on biharmonic maps from surfaces it simplifies enough (Proposition 5) to enable the use of a divergence argument (Theorem 6) and draw some consequences (Corollaries 8 and 9). However, the main consequences are for CMC biharmonic surfaces.

To close the article, we show that, in any ambient space, the condition of biharmonicity preserves the holomorphicity of the Hopf differential of CMC surfaces (Theorem 20).

Biharmonic CMC surfaces were also studied in [Fetcu and Pinheiro 2013; Ou and Wang 2011] and [Sasahara 2007].

The conventions we adopt are that the Riemann curvature tensor is

$$
R(X, Y)=\left[\nabla_{X}, \nabla_{Y}\right]-\nabla_{[X, Y]},
$$

while its $(0,4)$ counterpart is

$$
R(X, Y, Z, W)=\langle R(X, Y) W, Z\rangle .
$$

The choice of sign for the Laplacians on sections and functions is the same, and on the real line $\Delta f=-f^{\prime \prime}$.

All objects, unless specified, are smooth and we assume summation on repeated indices, when apt.

\section{The biharmonic stress-energy tensor on surfaces}

Since biharmonic maps stem from a variational problem, one can apply the general principle of studying the same functional but under variations of the domain metric. This idea taken up on the bienergy leads to the biharmonic stress-energy tensor, which is symmetric and of type $(0,2)$; see [Loubeau et al. 2008]. 
Definition 1. Let $(M, g)$ and $(N, h)$ be Riemannian manifolds and $\phi: M \rightarrow N$ a smooth map. The biharmonic stress-energy tensor of $\phi$ is

$$
S_{2}(X, Y)=\left\{\frac{|\tau(\phi)|^{2}}{2}+\langle d \phi, \nabla \tau(\phi)\rangle\right\} g(X, Y)-T(X, Y),
$$

where $T(X, Y)=\left\langle d \phi(X), \nabla_{Y} \tau(\phi)\right\rangle+\left\langle d \phi(Y), \nabla_{X} \tau(\phi)\right\rangle$.

The main feature of $S_{2}$ is satisfying Hilbert's principle of being divergence-free at critical points [Loubeau et al. 2008; Jiang 1987]; that is, $\operatorname{div} S_{2}=-\left\langle d \phi, \tau_{2}(\phi)\right\rangle$.

In order to exploit the biharmonicity of the map $\phi$, we compute the rough Laplacian of its biharmonic stress-energy tensor. This second-order operator on $(0,2)$-tensors will reveal curvature terms which combine with the bitension field, and formulas will involve swapping vector positions in the third fundamental form of $\phi$, with curvature appearing according to a lemma we quote separately, without proof.

Lemma 2. Let $\phi:\left(M^{m}, g\right) \rightarrow\left(N^{n}, h\right)$ be a smooth map. Then

$$
\left(\nabla^{2} d \phi\right)(X, Y, Z)-\left(\nabla^{2} d \phi\right)(Z, Y, X)=R(X, Z) d \phi(Y)-d \phi\left(R^{M}(X, Z) Y\right)
$$

for any $X, Y, Z \in C(T M)$.

Proposition 3 (the rough Laplacian of $\left.S_{2}\right)$. Let $(M, g)$ and $(N, h)$ be Riemannian manifolds and $\phi: M \rightarrow N$ a smooth map; then the (rough) Laplacian of $S_{2}$ is the symmetric $(0,2)$-tensor

$$
\begin{aligned}
\left(\Delta^{R} S_{2}\right)( & X, Y) \\
=(\langle\Delta & \tau(\phi), \tau(\phi)\rangle-2|\nabla \tau(\phi)|^{2}-2 \sum\left\langle R\left(X_{i}, X_{j}\right) d \phi\left(X_{i}\right), \nabla_{X_{j}} \tau(\phi)\right\rangle \\
& -2\left\langle d \phi\left(\operatorname{Ricci}^{M}(\cdot)\right), \nabla_{(\cdot)} \tau(\phi)\right\rangle-2\left\langle\nabla d \phi, \nabla^{2} \tau(\phi)\right\rangle+\langle d \phi, \nabla(\Delta \tau(\phi))\rangle \\
& -\left\langle\nabla\left(\operatorname{trace} R^{N}(d \phi(\cdot), \tau(\phi)) d \phi(\cdot)\right), d \phi\right\rangle \\
& \left.-\left\langle\operatorname{trace} R^{N}(d \phi(\cdot), \tau(\phi)) d \phi(\cdot), \tau(\phi)\right\rangle\right) g(X, Y) \\
+ & 2\left\langle\nabla_{X} \tau(\phi), \nabla_{Y} \tau(\phi)\right\rangle+\sum\left\langle R\left(X_{i}, X\right) d \phi\left(X_{i}\right), \nabla_{Y} \tau(\phi)\right\rangle \\
+ & \sum\left\langle R\left(X_{i}, Y\right) d \phi\left(X_{i}\right), \nabla_{X} \tau(\phi)\right\rangle+\left\langle d \phi\left(\operatorname{Ricci}^{M}(X)\right), \nabla_{Y} \tau(\phi)\right\rangle \\
+ & \left\langle d \phi\left(\operatorname{Ricci}^{M}(Y)\right), \nabla_{X} \tau(\phi)\right\rangle+2 \sum\left\langle\nabla d \phi\left(X_{i}, X\right),\left(\nabla^{2} \tau(\phi)\right)\left(X_{i}, Y\right)\right\rangle \\
+ & 2 \sum\left\langle\nabla d \phi\left(X_{i}, Y\right),\left(\nabla^{2} \tau(\phi)\right)\left(X_{i}, X\right)\right\rangle-\left\langle d \phi(X), \nabla_{Y}(\Delta \tau(\phi))\right\rangle \\
& -\left\langle d \phi(Y), \nabla_{X}(\Delta \tau(\phi))\right\rangle+\sum\left\langle d \phi(X), R\left(X_{i}, Y\right) \nabla_{X_{i}} \tau(\phi)\right\rangle \\
+ & \sum\left\langle d \phi(Y), R\left(X_{i}, X\right) \nabla_{X_{i}} \tau(\phi)\right\rangle+\sum\left\langle d \phi(X), \nabla_{X_{i}} R\left(X_{i}, Y\right) \tau(\phi)\right\rangle \\
+ & \sum\left\langle d \phi(Y), \nabla_{X_{i}} R\left(X_{i}, X\right) \tau(\phi)\right\rangle+\left\langle d \phi(X), \nabla_{\operatorname{Ricci}^{M}(Y)} \tau(\phi)\right\rangle \\
+ & \left\langle d \phi(Y), \nabla_{\operatorname{Ricci}^{M}(X)} \tau(\phi)\right\rangle,
\end{aligned}
$$

where $\left\{X_{i}\right\}$ is a geodesic frame around the point $p \in M$. 
Proof. Let $\phi:(M, g) \rightarrow(N, h)$ be a smooth map between Riemannian manifolds. We will work with a geodesic frame $\left\{X_{i}\right\}$ around the point $p \in M$ and evaluate at $p$.

Writing out the Laplacian in the geodesic frame yields $\Delta(\langle d \phi, \nabla \tau(\phi)\rangle)$

$$
\begin{array}{r}
=-\sum\left\{\left\langle\nabla_{X_{i}}\left[\nabla d \phi\left(X_{i}, X_{j}\right)\right], \nabla_{X_{j}} \tau(\phi)\right\rangle+2\left\langle\nabla d \phi\left(X_{i}, X_{j}\right),\left(\nabla^{2} \tau(\phi)\right)\left(X_{i}, X_{j}\right)\right\rangle\right. \\
\left.+\left\langle d \phi\left(X_{j}\right), \nabla_{X_{i}} \nabla_{X_{i}} \nabla_{X_{j}} \tau(\phi)\right\rangle-\left\langle d \phi\left(X_{j}\right), \nabla_{X_{i}} \nabla_{\nabla_{X_{i}}} X_{j} \tau(\phi)\right\rangle\right\},
\end{array}
$$

and by the symmetry formula of the third fundamental form, we have

$$
\sum \nabla_{X_{i}}\left[\nabla d \phi\left(X_{i}, X_{j}\right)\right]=\nabla_{X_{j}} \tau(\phi)+\sum R\left(X_{i}, X_{j}\right) d \phi\left(X_{i}\right)+d \phi\left(\operatorname{Ricci}^{M}\left(X_{j}\right)\right),
$$

and

$$
\begin{aligned}
\sum\left(\nabla_{X_{i}} \nabla_{X_{i}} \nabla_{X_{j}} \tau(\phi)\right. & \left.-\nabla_{X_{i}} \nabla_{\nabla_{X_{i}} X_{j}} \tau(\phi)\right) \\
=\sum\left\{\nabla_{X_{j}} \nabla_{X_{i}} \nabla_{X_{i}} \tau(\phi)+\nabla_{\left[X_{i}, X_{j}\right]} \nabla_{X_{i}} \tau(\phi)+R\left(X_{i}, X_{j}\right) \nabla_{X_{i}} \tau(\phi)\right. & \\
& +\nabla_{X_{i}} R\left(X_{i}, X_{j}\right) \tau(\phi) \\
& \left.-\left(\nabla_{\nabla_{X_{j}} X_{i}} \nabla_{X_{i}} \tau(\phi)+\nabla_{\left[X_{i}, \nabla_{X_{j}} X_{i}\right]} \tau(\phi)+R\left(X_{i}, \nabla_{X_{j}} X_{i}\right) \tau(\phi)\right)\right\} \\
=-\nabla_{X_{j}}(\Delta \tau(\phi))+\sum\left\{\left(\nabla^{2} \tau(\phi)\right)\left(X_{j}, \nabla_{X_{i}} X_{i}\right)\right. & +R\left(X_{i}, X_{j}\right) \nabla_{X_{i}} \tau(\phi) \\
+ & \left.+\nabla_{X_{i}} R\left(X_{i}, X_{j}\right) \tau(\phi)\right\} \\
+ &
\end{aligned}
$$

since

$$
\sum\left[X_{i}, \nabla_{X_{j}} X_{i}\right]=\sum \nabla_{X_{j}} \nabla_{X_{i}} X_{i}-\operatorname{Ricci}^{M}\left(X_{j}\right)
$$

Therefore

$\Delta(\langle d \phi, \nabla \tau(\phi)\rangle)$

$$
\begin{aligned}
=- & \sum\left\langle\nabla_{X_{j}} \tau(\phi), \nabla_{X_{j}} \tau(\phi)\right\rangle-\sum\left\langle R\left(X_{i}, X_{j}\right) d \phi\left(X_{i}\right), \nabla_{X_{j}} \tau(\phi)\right\rangle \\
& -\sum\left\langle d \phi\left(\operatorname{Ricci}^{M}\left(X_{j}\right)\right), \nabla_{X_{j}} \tau(\phi)\right\rangle \\
- & 2\left\langle\nabla d \phi, \nabla^{2} \tau(\phi)\right\rangle+\sum\left\langle d \phi\left(X_{j}\right), \nabla_{X_{j}}(\Delta \tau(\phi))\right\rangle \\
- & \sum\left(\left\langle d \phi\left(X_{j}\right), R\left(X_{i}, X_{j}\right) \nabla_{X_{i}}(\tau(\phi))\right\rangle+\right. \\
& \left\langle d \phi\left(X_{j}\right), \nabla_{X_{i}} R\left(X_{i}, X_{j}\right) \tau(\phi)\right\rangle \\
& \left.+\left\langle d \phi\left(X_{j}\right), \nabla_{\operatorname{Ricci}^{M}\left(X_{j}\right)} \tau(\phi)\right\rangle\right),
\end{aligned}
$$

but

$\sum\left\langle d \phi\left(X_{j}\right), \nabla_{X_{i}} R\left(X_{i}, X_{j}\right) \tau(\phi)\right\rangle$

$$
=\sum X_{i}\left\langle\operatorname{trace} R^{N}(d \phi(\cdot), \tau(\phi)) d \phi(\cdot), d \phi\left(X_{i}\right)\right\rangle-\langle\nabla d \phi, R(\cdot, \cdot) \tau(\phi)\rangle
$$$$
=\left\langle\nabla\left(\operatorname{trace} R^{N}(d \phi(\cdot), \tau(\phi)) d \phi(\cdot)\right), d \phi\right\rangle
$$$$
+\left\langle\operatorname{trace} R^{N}(d \phi(\cdot), \tau(\phi)) d \phi(\cdot), \tau(\phi)\right\rangle,
$$ 
and

$$
\sum\left\langle R\left(X_{i}, X_{j}\right) d \phi\left(X_{i}\right), \nabla_{X_{j}} \tau(\phi)\right\rangle=\sum\left\langle R\left(X_{i}, X_{j}\right) \nabla_{X_{i}} \tau(\phi), d \phi\left(X_{j}\right)\right\rangle,
$$

whilst

$$
\sum\left\langle d \phi\left(\operatorname{Ricci}^{M}(\cdot)\right), \nabla \tau(\phi)\right\rangle=\sum\left\langle d \phi(\cdot), \nabla_{\operatorname{Ricci}^{M}(\cdot)} \tau(\phi)\right\rangle,
$$

so the Laplacian of the scalar term is

$$
\begin{aligned}
\Delta\left(\frac{|\tau(\phi)|^{2}}{2}\right. & +\langle d \phi, \nabla \tau(\phi)\rangle) \\
= & \langle\Delta \tau(\phi), \tau(\phi)\rangle-2|\nabla \tau(\phi)|^{2} \\
& -2 \sum\left\langle R\left(X_{i}, X_{j}\right) d \phi\left(X_{i}\right), \nabla_{X_{j}} \tau(\phi)\right\rangle-2\left\langle d \phi\left(\operatorname{Ricci}^{M}(\cdot)\right), \nabla_{(\cdot)} \tau(\phi)\right\rangle \\
& -2\left\langle\nabla d \phi, \nabla^{2} \tau(\phi)\right\rangle+\left\langle d \phi(\cdot), \nabla_{(\cdot)}(\Delta \tau(\phi))\right\rangle \\
& -\left\langle\nabla\left(\operatorname{trace} R^{N}(d \phi(\cdot), \tau(\phi)) d \phi(\cdot)\right), d \phi\right\rangle \\
& -\left\langle\operatorname{trace} R^{N}(d \phi(\cdot), \tau(\phi)) d \phi(\cdot), \tau(\phi)\right\rangle .
\end{aligned}
$$

On the other hand, to compute the (rough) Laplacian of the symmetric two-tensor

$$
T(X, Y)=\left\langle d \phi(X), \nabla_{Y} \tau(\phi)\right\rangle+\left\langle d \phi(Y), \nabla_{X} \tau(\phi)\right\rangle,
$$

we put $X=X_{k}$ and $Y=X_{j}$ and obtain, still evaluating expressions at the point $p$,

$$
\begin{aligned}
-\left(\Delta^{R} T\right)(X, Y)=\sum & \left(\left\langle\nabla_{X_{i}} \nabla_{X_{i}} d \phi(X), \nabla_{Y} \tau(\phi)\right\rangle+2\left\langle\nabla_{X_{i}} d \phi(X), \nabla_{X_{i}} \nabla_{Y} \tau(\phi)\right\rangle\right. \\
+ & \left\langle d \phi(X), \nabla_{X_{i}} \nabla_{X_{i}} \nabla_{Y} \tau(\phi)\right\rangle+\left\langle\nabla_{X_{i}} \nabla_{X_{i}} d \phi(Y), \nabla_{X} \tau(\phi)\right\rangle \\
+ & 2\left\langle\nabla_{X_{i}} d \phi(Y), \nabla_{X_{i}} \nabla_{X} \tau(\phi)\right\rangle+\left\langle d \phi(Y), \nabla_{X_{i}} \nabla_{X_{i}} \nabla_{X} \tau(\phi)\right\rangle \\
& \left.-\left\langle d \phi(Y), \nabla_{X_{i}} \nabla_{\nabla_{X_{i}} X} \tau(\phi)\right\rangle-\left\langle d \phi(X), \nabla_{X_{i}} \nabla_{\nabla_{X_{i}}} \tau(\phi)\right\rangle\right),
\end{aligned}
$$

since $\nabla_{X_{i}} \nabla_{X_{i}} X_{j}$ vanishes at the point $p$. This last expression simplifies further if we use the symmetries properties of the third fundamental form of $\phi$ to obtain

$$
\begin{aligned}
\sum \nabla_{X_{i}} \nabla_{X_{i}} d \phi(X) & =\sum\left(\nabla^{2} d \phi\right)\left(X_{i}, X_{i}, X\right) \\
& =\nabla_{X} \tau(\phi)+\sum R\left(X_{i}, X\right) d \phi\left(X_{i}\right)+d \phi\left(\operatorname{Ricci}^{M}(X)\right),
\end{aligned}
$$

and the curvature tensor of the pullback bundle for

$$
\begin{aligned}
\sum\left(\left\langle d \phi(X), \nabla_{X_{i}} \nabla_{X_{i}} \nabla_{Y} \tau(\phi)\right\rangle-\left\langle d \phi(X), \nabla_{X_{i}} \nabla_{\nabla_{X_{i}}} \tau(\phi)\right\rangle\right) & \\
= & -\left\langle d \phi(X), \nabla_{Y}(\Delta \tau(\phi))\right\rangle+\sum\left\langle d \phi(X), R\left(X_{i}, Y\right) \nabla_{X_{i}} \tau(\phi)\right\rangle \\
& +\sum\left\langle d \phi(X), \nabla_{X_{i}} R\left(X_{i}, Y\right) \tau(\phi)\right\rangle+\left\langle d \phi(X), \nabla_{\operatorname{Ricci}^{M}(Y)} \tau(\phi)\right\rangle .
\end{aligned}
$$


The Laplacian of the tensor $T$ is then equal to

$$
\begin{aligned}
-\left(\Delta^{R} T\right)(X, & Y) \\
= & \left\langle\nabla_{X} \tau(\phi), \nabla_{Y} \tau(\phi)\right\rangle+\sum\left\langle R\left(X_{i}, X\right) d \phi\left(X_{i}\right), \nabla_{Y} \tau(\phi)\right\rangle \\
& +\left\langle d \phi\left(\operatorname{Ricci}^{M}(X)\right), \nabla_{Y} \tau(\phi)\right\rangle+2 \sum\left\langle\nabla d \phi\left(X_{i}, X\right),\left(\nabla^{2} \tau(\phi)\right)\left(X_{i}, Y\right)\right\rangle \\
& -\left\langle d \phi(X), \nabla_{Y}(\Delta \tau(\phi))\right\rangle+\sum\left\langle d \phi(X), R\left(X_{i}, Y\right) \nabla_{X_{i}} \tau(\phi)\right\rangle \\
& +\sum\left\langle d \phi(X), \nabla_{X_{i}} R\left(X_{i}, Y\right) \tau(\phi)\right\rangle+\left\langle d \phi(X), \nabla_{\operatorname{Ricci}^{M}(Y)} \tau(\phi)\right\rangle \\
& +\left\langle\nabla_{Y} \tau(\phi), \nabla_{X} \tau(\phi)\right\rangle+\sum\left\langle R\left(X_{i}, Y\right) d \phi\left(X_{i}\right), \nabla_{X} \tau(\phi)\right\rangle \\
& +\left\langle d \phi\left(\operatorname{Ricci}^{M}(Y)\right), \nabla_{X} \tau(\phi)\right\rangle+2 \sum\left\langle\nabla d \phi\left(X_{i}, Y\right),\left(\nabla^{2} \tau(\phi)\right)\left(X_{i}, X\right)\right\rangle \\
& -\left\langle d \phi(Y), \nabla_{X}(\Delta \tau(\phi))\right\rangle+\sum\left\langle d \phi(Y), R\left(X_{i}, X\right) \nabla_{X_{i}} \tau(\phi)\right\rangle \\
& +\sum\left\langle d \phi(Y), \nabla_{X_{i}} R\left(X_{i}, X\right) \tau(\phi)\right\rangle+\left\langle d \phi(Y), \nabla_{\operatorname{Ricci}^{M}(X)} \tau(\phi)\right\rangle .
\end{aligned}
$$

Summing the various parts together yields the proposition.

Remark 4. In order to see the geometric meaning of the term $\sum \nabla_{X_{i}} R\left(X_{i}, X\right) \tau(\phi)$, we can rewrite it as $\sum(\nabla R)\left(X_{i}, X_{i}, X, \tau(\phi)\right)+\sum R\left(X_{i}, X\right) \nabla_{X_{i}} \tau(\phi)$.

While the general expression for the rough Laplacian of $S_{2}$ at first seems unwieldy, in a manner reminiscent of its harmonic counterpart (see [Baird et al. 2011]) it becomes amenable when the domain is a surface and the map biharmonic. The final formula only involves three ingredients: the tensor $S_{2}$ itself, the Gaussian curvature and the norm of the tension field of the map. This paves the way for a series of propositions and corollaries for both maps and Riemannian immersions, where topological and curvature conditions restrict the existence of biharmonic maps.

Proposition 5. Let $\phi:\left(M^{2}, g\right) \rightarrow(N, h)$ be a biharmonic map defined on a surface $M^{2}$. The Laplacian of its biharmonic stress-energy tensor is

$$
\Delta^{R} S_{2}=-2 K^{M} S_{2}+\nabla d\left(|\tau(\phi)|^{2}\right)+\left\{K^{M}|\tau(\phi)|^{2}+\Delta|\tau(\phi)|^{2}\right\} g,
$$

where $K^{M}$ is the Gaussian curvature of $\left(M^{2}, g\right)$.

Proof. Since $\operatorname{dim} M=2$, its Ricci curvature is $\operatorname{Ricci}^{M}=K^{M} I$, with $K^{M} \in C^{\infty}(M)$. We will work with a geodesic frame $\left\{X_{1}, X_{2}\right\}$ around a point $p \in M^{2}$ and evaluate all expressions at this point.

As $\Delta^{R} S_{2}$ is a symmetric $(0,2)$-tensor, there are only two cases to consider, and, from the previous proposition, combined with basic symmetries of the curvature tensor and the biharmonicity condition, we have 
$\left(\Delta^{R} S_{2}\right)\left(X_{1}, X_{2}\right)$

$=2\left\langle\nabla_{X_{1}} \tau(\phi), \nabla_{X_{2}} \tau(\phi)\right\rangle-\left\langle d \phi\left(X_{2}\right), \nabla_{X_{1}}(\Delta \tau(\phi))\right\rangle$

$+2 K^{M}\left\{\left\langle d \phi\left(X_{1}\right), \nabla_{X_{2}} \tau(\phi)\right\rangle+\left\langle d \phi\left(X_{2}\right), \nabla_{X_{1}} \tau(\phi)\right\rangle\right\}$

$+2\left\langle\nabla d \phi\left(X_{1}, X_{2}\right),-\Delta \tau(\phi)\right\rangle+2\left\langle\nabla d \phi\left(X_{1}, X_{1}\right),\left(\nabla^{2} \tau(\phi)\right)\left(X_{1}, X_{2}\right)\right\rangle$

$+2\left\langle\nabla d \phi\left(X_{2}, X_{2}\right),\left(\nabla^{2} \tau(\phi)\right)\left(X_{2}, X_{1}\right)\right\rangle-\left\langle d \phi\left(X_{1}\right), \nabla_{X_{2}}(\Delta \tau(\phi))\right\rangle$

$+\left\langle d \phi\left(X_{1}\right), \nabla_{X_{1}} R\left(X_{1}, X_{2}\right) \tau(\phi)\right\rangle+\left\langle d \phi\left(X_{2}\right), \nabla_{X_{2}} R\left(X_{2}, X_{1}\right) \tau(\phi)\right\rangle$

$=2\left\langle\nabla_{X_{1}} \tau(\phi), \nabla_{X_{2}} \tau(\phi)\right\rangle+2 K^{M}\left\{\left\langle d \phi\left(X_{1}\right), \nabla_{X_{2}} \tau(\phi)\right\rangle+\left\langle d \phi\left(X_{2}\right), \nabla_{X_{1}} \tau(\phi)\right\rangle\right\}$

$+2\left\langle\nabla d \phi\left(X_{1}, X_{1}\right),\left(\nabla^{2} \tau(\phi)\right)\left(X_{1}, X_{2}\right)\right\rangle+2\left\langle\nabla d \phi\left(X_{2}, X_{2}\right),\left(\nabla^{2} \tau(\phi)\right)\left(X_{2}, X_{1}\right)\right\rangle$

$-\left\langle\nabla_{X_{1}} d \phi\left(X_{1}\right), R\left(X_{1}, X_{2}\right) \tau(\phi)\right\rangle-\left\langle\nabla_{X_{2}} d \phi\left(X_{2}\right), R\left(X_{2}, X_{1}\right) \tau(\phi)\right\rangle$.

But

$2\left\langle\nabla d \phi\left(X_{1}, X_{1}\right),\left(\nabla^{2} \tau(\phi)\right)\left(X_{1}, X_{2}\right)\right\rangle-\left\langle\nabla d \phi\left(X_{1}, X_{1}\right), R\left(X_{1}, X_{2}\right) \tau(\phi)\right\rangle$

$$
=\left\langle\nabla d \phi\left(X_{1}, X_{1}\right), 2 \nabla_{X_{1}} \nabla_{X_{2}} \tau(\phi)-\nabla_{X_{1}} \nabla_{X_{2}} \tau(\phi)+\nabla_{X_{2}} \nabla_{X_{1}} \tau(\phi)\right\rangle,
$$

so

$\left(\Delta^{R} S_{2}\right)\left(X_{1}, X_{2}\right)=2 K^{M}\left\{\left\langle d \phi\left(X_{1}\right), \nabla_{X_{2}} \tau(\phi)\right\rangle+\left\langle d \phi\left(X_{2}\right), \nabla_{X_{1}} \tau(\phi)\right\rangle\right\}$

$+2\left\langle\nabla_{X_{1}} \tau(\phi), \nabla_{X_{2}} \tau(\phi)\right\rangle+\left\langle\tau(\phi), \nabla_{X_{1}} \nabla_{X_{2}} \tau(\phi)+\nabla_{X_{2}} \nabla_{X_{1}} \tau(\phi)\right\rangle$.

Since

$\left(\nabla d|\tau(\phi)|^{2}\right)\left(X_{1}, X_{2}\right)$

$$
=\left\langle\nabla_{X_{1}} \nabla_{X_{2}} \tau(\phi)+\nabla_{X_{2}} \nabla_{X_{1}} \tau(\phi), \tau(\phi)\right\rangle+2\left\langle\nabla_{X_{1}} \tau(\phi), \nabla_{X_{2}} \tau(\phi)\right\rangle,
$$

we deduce that

$$
\left(\Delta^{R} S_{2}\right)\left(X_{1}, X_{2}\right)=-2 K^{M} S_{2}\left(X_{1}, X_{2}\right)+\left(\nabla d|\tau(\phi)|^{2}\right)\left(X_{1}, X_{2}\right) .
$$

The other case to look at is when the two vectors are the same, and then Proposition 3 shows that, using the symmetries of $R^{N}$,

$\left(\Delta^{R} S_{2}\right)\left(X_{1}, X_{1}\right)$

$=-2\left\langle R^{N}\left(d \phi\left(X_{1}\right), \tau(\phi)\right) d \phi\left(X_{1}\right), \tau(\phi)\right\rangle$

$-2\left\langle R^{N}\left(d \phi\left(X_{2}\right), \tau(\phi)\right) d \phi\left(X_{2}\right), \tau(\phi)\right\rangle-2\left\langle\nabla_{X_{2}} \tau(\phi), \nabla_{X_{2}} \tau(\phi)\right\rangle$

$-2 K^{M}\left\langle d \phi\left(X_{2}\right), \nabla_{X_{2}} \tau(\phi)\right\rangle-2\left\langle\nabla d \phi\left(X_{2}, X_{2}\right),\left(\nabla^{2} \tau(\phi)\right)\left(X_{2}, X_{2}\right)\right\rangle$

$-2\left\langle\nabla d \phi\left(X_{1}, X_{2}\right),\left(\nabla^{2} \tau(\phi)\right)\left(X_{1}, X_{2}\right)\right\rangle+2\left\langle d \phi\left(X_{1}\right), \nabla_{X_{1}}(\Delta \tau(\phi))\right\rangle$

$+2\left\langle d \phi\left(X_{2}\right), \nabla_{X_{2}}(\Delta \tau(\phi))\right\rangle+2 K^{M}\left\langle d \phi\left(X_{1}\right), \nabla_{X_{1}} \tau(\phi)\right\rangle$

$+2\left\langle\nabla d \phi\left(X_{1}, X_{1}\right),\left(\nabla^{2} \tau(\phi)\right)\left(X_{1}, X_{1}\right)\right\rangle+2\left\langle\nabla d \phi\left(X_{2}, X_{1}\right),\left(\nabla^{2} \tau(\phi)\right)\left(X_{2}, X_{1}\right)\right\rangle$

$-2\left\langle d \phi\left(X_{1}\right), \nabla_{X_{1}}(\Delta \tau(\phi))\right\rangle+2\left\langle d \phi\left(X_{1}\right), \nabla_{X_{2}} R\left(X_{2}, X_{1}\right) \tau(\phi)\right\rangle$ 


$$
\begin{aligned}
=- & \left|\nabla_{X_{2}} \tau(\phi)\right|^{2}-2 K^{M}\left\langle d \phi\left(X_{2}\right), \nabla_{X_{2}} \tau(\phi)\right\rangle+2 K^{M}\left\langle d \phi\left(X_{1}\right), \nabla_{X_{1}} \tau(\phi)\right\rangle \\
& -2\left\langle R^{N}\left(d \phi\left(X_{1}\right), \tau(\phi)\right) d \phi\left(X_{1}\right), \nabla d \phi\left(X_{1}, X_{1}\right)\right\rangle \\
& -2 X_{2}\left\langle d \phi\left(X_{2}\right), R^{N}\left(d \phi\left(X_{1}\right), \tau(\phi)\right) d \phi\left(X_{1}\right)\right\rangle \\
& -2\left\langle R^{N}\left(d \phi\left(X_{2}\right), \tau(\phi)\right) d \phi\left(X_{2}\right), \nabla d \phi\left(X_{1}, X_{1}\right)\right\rangle \\
& -2\left\langle\nabla d \phi\left(X_{2}, X_{2}\right),\left(\nabla^{2} \tau(\phi)\right)\left(X_{2}, X_{2}\right)\right\rangle \\
+ & 2\left\langle\nabla d \phi\left(X_{1}, X_{1}\right),\left(\nabla^{2} \tau(\phi)\right)\left(X_{1}, X_{1}\right)\right\rangle-2\left\langle\nabla d \phi\left(X_{1}, X_{2}\right), R\left(X_{1}, X_{2}\right) \tau(\phi)\right\rangle \\
+ & 2\left\langle d \phi\left(X_{1}\right), \nabla_{X_{2}} R\left(X_{2}, X_{1}\right) \tau(\phi)\right\rangle,
\end{aligned}
$$

since

i) $-2\left\langle\nabla d \phi\left(X_{1}, X_{2}\right),\left(\nabla^{2} \tau(\phi)\right)\left(X_{1}, X_{2}\right)\right.$

$+2\left\langle\nabla d \phi\left(X_{2}, X_{1}\right),\left(\nabla^{2} \tau(\phi)\right)\left(X_{2}, X_{1}\right)\right\rangle$

$$
=-2\left\langle\nabla d \phi\left(X_{1}, X_{2}\right), R\left(X_{1}, X_{2}\right) \tau(\phi)\right\rangle,
$$

ii) $-2\left\langle R^{N}\left(d \phi\left(X_{1}\right), \tau(\phi)\right) d \phi\left(X_{1}\right), \tau(\phi)\right\rangle$

$$
-2\left\langle d \phi\left(X_{2}\right), \nabla_{X_{2}} R^{N}\left(d \phi\left(X_{1}\right), \tau(\phi)\right) d \phi\left(X_{1}\right)\right\rangle
$$

$$
\begin{aligned}
=- & \left\langle R^{N}\left(d \phi\left(X_{1}\right), \tau(\phi)\right) d \phi\left(X_{1}\right), \nabla_{X_{1}} d \phi\left(X_{1}\right)\right\rangle \\
& -2\left\langle R^{N}\left(d \phi\left(X_{1}\right), \tau(\phi)\right) d \phi\left(X_{1}\right), \nabla_{X_{2}} d \phi\left(X_{2}\right)\right\rangle \\
& -2 X_{2}\left\langle d \phi\left(X_{2}\right), R^{N}\left(d \phi\left(X_{1}\right), \tau(\phi)\right) d \phi\left(X_{1}\right)\right\rangle \\
& +2\left\langle\nabla_{X_{2}} d \phi\left(X_{2}\right), R^{N}\left(d \phi\left(X_{1}\right), \tau(\phi)\right) d \phi\left(X_{1}\right)\right\rangle,
\end{aligned}
$$

iii) $-2\left\langle R^{N}\left(d \phi\left(X_{2}\right), \tau(\phi)\right) d \phi\left(X_{2}\right), \tau(\phi)\right\rangle$

$-2\left\langle d \phi\left(X_{2}\right), \nabla_{X_{2}} R^{N}\left(d \phi\left(X_{2}\right), \tau(\phi)\right) d \phi\left(X_{2}\right)\right\rangle$

$$
=-2\left\langle R^{N}\left(d \phi\left(X_{2}\right), \tau(\phi)\right) d \phi\left(X_{2}\right), \nabla d \phi\left(X_{1}, X_{1}\right)\right\rangle .
$$

Observe that

$$
\begin{aligned}
& -X_{2}\left\langle d \phi\left(X_{2}\right), R^{N}\left(d \phi\left(X_{1}\right), \tau(\phi)\right) d \phi\left(X_{1}\right)\right\rangle+\left\langle d \phi\left(X_{1}\right), \nabla_{X_{2}} R\left(X_{2}, X_{1}\right) \tau(\phi)\right\rangle \\
& =-X_{2} R^{N}\left(d \phi\left(X_{2}\right), d \phi\left(X_{1}\right), d \phi\left(X_{1}\right), \tau(\phi)\right) \\
& \quad+X_{2} R^{N}\left(d \phi\left(X_{2}\right), d \phi\left(X_{1}\right), d \phi\left(X_{1}\right), \tau(\phi)\right)+\left\langle\nabla d \phi\left(X_{1}, X_{2}\right), R\left(X_{1}, X_{2}\right) \tau(\phi)\right\rangle \\
& \text { so } \\
& \left(\Delta^{R} S_{2}\right)\left(X_{1}, X_{1}\right) \\
& =-2\left|\nabla_{X_{2}} \tau(\phi)\right|^{2}-2 K^{M}\left\langle d \phi\left(X_{2}\right), \nabla_{X_{2}} \tau(\phi)\right\rangle \\
& \quad+2 K^{M}\left\langle d \phi\left(X_{1}\right), \nabla_{X_{1}} \tau(\phi)\right\rangle-2\left\langle R^{N}\left(d \phi\left(X_{1}\right), \tau(\phi)\right) d \phi\left(X_{1}\right), \nabla d \phi\left(X_{1}, X_{1}\right)\right\rangle \\
& \quad+2\left\langle\nabla d \phi\left(X_{1}, X_{1}\right),\left(\nabla^{2} \tau(\phi)\right)\left(X_{1}, X_{1}\right)\right\rangle \\
& \quad-2\left\langle R^{N}\left(d \phi\left(X_{2}\right), \tau(\phi)\right) d \phi\left(X_{2}\right), \nabla d \phi\left(X_{1}, X_{1}\right)\right\rangle \\
& \quad-2\left\langle\nabla d \phi\left(X_{2}, X_{2}\right),\left(\nabla^{2} \tau(\phi)\right)\left(X_{2}, X_{2}\right)\right\rangle .
\end{aligned}
$$


But

$$
\left\langle\tau(\phi), \nabla_{X_{2}} \nabla_{X_{2}} \tau(\phi)\right\rangle=-\frac{1}{2} \Delta|\tau(\phi)|^{2}-\frac{1}{2} X_{1} X_{1}\left(|\tau(\phi)|^{2}\right)-\left|\nabla_{X_{2}} \tau(\phi)\right|^{2},
$$

so

$$
\begin{aligned}
\left(\Delta^{R} S_{2}\right)\left(X_{1}, X_{1}\right)=- & 2 K^{M} S_{2}\left(X_{1}, X_{1}\right)+\left\{K^{M}|\tau(\phi)|^{2}+\Delta|\tau(\phi)|^{2}\right\} g\left(X_{1}, X_{1}\right) \\
& +\left(\nabla d|\tau(\phi)|^{2}\right)\left(X_{1}, X_{1}\right),
\end{aligned}
$$

with a similar expression for $\left(\Delta^{R} S_{2}\right)\left(X_{2}, X_{2}\right)$. Therefore

$$
\Delta^{R} S_{2}=-2 K^{M} S_{2}+\nabla d\left(|\tau(\phi)|^{2}\right)+\left\{K^{M}|\tau(\phi)|^{2}+\Delta|\tau(\phi)|^{2}\right\} g .
$$

The expression for the Laplacian of the biharmonic stress-energy tensor on a surface is simple enough to be contracted with $S_{2}$ itself and combined with the divergence theorem, if the domain is assumed to be compact. The ensuing integral formula tightly binds the tensor $S_{2}$, the Gaussian curvature and the norm of the tension field together, and conditions on two of them determine the third.

More geometrical applications will be found for Riemannian immersions in the next section.

Theorem 6. Let $\phi: M^{2} \rightarrow N^{n}$ be a biharmonic map and assume $M^{2}$ is compact. Then

$$
\int_{M}\left|\nabla S_{2}\right|^{2} v_{g}+2 \int_{M} K^{M}\left(\left|S_{2}\right|^{2}-\frac{|\tau(\phi)|^{4}}{2}\right) v_{g}=\int_{M}\left|d\left(|\tau(\phi)|^{2}\right)\right|^{2} v_{g},
$$

where $K^{M}$ is the Gaussian curvature of $\left(M^{2}, g\right)$.

Proof. Observe that

$$
\operatorname{div}\left\langle S_{2}, d\left(|\tau(\phi)|^{2}\right)\right\rangle=\left\langle\operatorname{div} S_{2}, d\left(|\tau(\phi)|^{2}\right)\right\rangle+\left\langle S_{2}, \operatorname{Hess}\left(|\tau(\phi)|^{2}\right)\right\rangle .
$$

As $\operatorname{div} S_{2}=0$, we have

$$
\int_{M}\left\langle S_{2}, \operatorname{Hess}\left(|\tau(\phi)|^{2}\right)\right\rangle v_{g}=\int_{M} \operatorname{div}\left\{\left\langle S_{2}, d\left(|\tau(\phi)|^{2}\right)\right\rangle\right\} v_{g}=0,
$$

which combined with the classical equality

$$
\int_{M}\left\langle\Delta^{R} S_{2}, S_{2}\right\rangle v_{g}=\int_{M}\left|\nabla S_{2}\right|^{2} v_{g}
$$

gives the theorem.

Remark 7. Note that the term $2\left|S_{2}\right|^{2}-|\tau(\phi)|^{4}$ is always nonnegative since it is equal to $\left(S_{2}\left(X_{1}, X_{1}\right)-S_{2}\left(X_{2}, X_{2}\right)\right)^{2}+4 S_{2}^{2}\left(X_{1}, X_{2}\right)$, and $\left|S_{2}\right|^{2}=|\tau(\phi)|^{4} / 2$ if and only if $S_{2}=|\tau(\phi)|^{2} g / 2$. 
A biharmonic map with parallel stress-energy tensor must have a tension field of constant norm [Loubeau et al. 2008], but Proposition 5 shows greater restrictions for two-dimensional domains.

Corollary 8. Let $\phi: M^{2} \rightarrow N^{n}$ be a biharmonic map, and assume $M$ is compact and $\nabla S_{2}=0$. Then $|\tau(\phi)|$ is constant and $\int_{M} K^{M} v_{g}=0$ or $S_{2}=|\tau(\phi)|^{2} g / 2$.

Proof. If $\nabla S_{2}=0$, then its norm and trace, $|\tau(\phi)|^{2}$, are constant, hence

$$
\left(\left|S_{2}\right|^{2}-\frac{|\tau(\phi)|^{4}}{2}\right) \int_{M} K^{M} v_{g}=0
$$

If the norm of the tension field is constant, we can deduce a partial converse for nonnegative curvature.

Corollary 9. Let $\phi:\left(M^{2}, g\right) \rightarrow\left(N^{n}, h\right)$ be a proper -biharmonic map with $|\tau(\phi)|^{2}$ constant. Assume $M$ is compact and $K^{M} \geq 0$. Then $S_{2}$ is parallel and $M$ is flat or $S_{2}=|\tau(\phi)|^{2} g / 2$.

\section{Constant mean curvature surfaces}

To be able to offer conditions with greater geometrical content, we concentrate our applications on Riemannian immersions. The recurrent condition on the map is pseudoumbilicity, as an equality between the shape operator $A_{H}$ in the direction of the mean curvature vector field $H$ and the metric.

The pivotal role of pseudoumbilical immersions, already observed in the study of the biharmonic stress-energy tensor (see [Loubeau et al. 2008]), emerges again in connection with the curvature of the domain surface, sometimes to the extent of determining its topology.

In the absence of compactness, the divergence theorem is substituted with a parabolicity argument on constant mean curvature surfaces, associated with a bound on the curvature tensor of the target space.

Finally, working with complex coordinates on a Riemann surface, the $(2,0)$-part of the $H$-component of the second fundamental form $B$ is shown to be holomorphic if and only if the mean curvature is constant.

Recall that if $\phi: M^{2} \rightarrow N$ is a pseudoumbilical proper-biharmonic Riemannian immersion then it is CMC. As a consequence, and since $S_{2}=-2|H|^{2} g+4 A_{H}$, a rewording of Corollaries 8 and 9 is as follows:

Corollary 10. Let $\phi:\left(M^{2}, g\right) \rightarrow\left(N^{n}, h\right)$ be a proper-biharmonic Riemannian immersion from a compact oriented surface, with $\nabla A_{H}=0$. Then $M$ is topologically a torus or pseudoumbilical. 
Corollary 11. Let $\phi:\left(M^{2}, g\right) \rightarrow\left(N^{n}, h\right)$ be a CMC proper-biharmonic Riemannian immersion. Assume $M$ is compact and $K^{M} \geq 0$. Then $\nabla A_{H}=0$ and $M$ is flat or pseudoumbilical.

The next result shows that pseudoumbilical points allow some flexibility of the curvature; since away from these points special coordinates exist in which the metric is conformally flat (with a globally defined factor), the shape operator has a simple expression, while its eigenvalues can be computed from the mean curvature vector field (see [Hasanis and Vlachos 1996] for a similar result).

Theorem 12. Let $\phi:\left(M^{2}, g\right) \rightarrow\left(N^{n}, h\right)$ be a CMC proper-biharmonic Riemannian immersion. We denote by $\lambda_{1}$ and $\lambda_{2}$ the principal curvatures of $M$ corresponding to $A_{H}$, with $\lambda_{1} \geq \lambda_{2}$, and let $\mu=\lambda_{1}-\lambda_{2}$. Consider $p \in M$ such that $\mu(p)>0$; that is, $p$ is a nonpseudoumbilical point. Then, around $p$ there is a local chart $(U ; x, y)$ which is both isothermal and a line of curvature coordinate system for $A_{H}$. We have, on $U$,

$$
\begin{gathered}
g=\frac{1}{\mu}\left(d x^{2}+d y^{2}\right), \quad\left\langle A_{H}(\cdot), \cdot\right\rangle=\frac{1}{\mu}\left(\lambda_{1} d x^{2}+\lambda_{2} d y^{2}\right), \\
\sum_{i=1}^{2} R^{N}\left(X_{i}, H, X_{i}, H\right)-\left|\nabla^{\perp} H\right|^{2}-2|H|^{4}>0,
\end{gathered}
$$

and

$$
\begin{aligned}
& \lambda_{1}=|H|^{2}+\frac{\sqrt{2}}{2} \sqrt{\sum_{i=1}^{2} R^{N}\left(X_{i}, H, X_{i}, H\right)-\left|\nabla^{\perp} H\right|^{2}-2|H|^{4}}, \\
& \lambda_{2}=|H|^{2}-\frac{\sqrt{2}}{2} \sqrt{\sum_{i=1}^{2} R^{N}\left(X_{i}, H, X_{i}, H\right)-\left|\nabla^{\perp} H\right|^{2}-2|H|^{4}},
\end{aligned}
$$

with $X_{1}=\sqrt{\mu} \partial x, X_{2}=\sqrt{\mu} \partial y$. Moreover

$$
\Delta \ln \left(\sum_{i=1}^{2} R^{N}\left(X_{i}, H, X_{i}, H\right)-\left|\nabla^{\perp} H\right|^{2}-2|H|^{4}\right)=-4 K^{M},
$$

and, in codimension one, the Gauss equation becomes

$$
\operatorname{Riem}^{N}\left(X_{1}, X_{2}\right)=K^{M}-2|H|^{2}+\frac{1}{2|H|^{2}} \operatorname{Ricci}^{N}(H, H) .
$$

Proof. Let $\lambda_{1}$ and $\lambda_{2}$ be the principal curvatures in the direction of $H$; that is, $\lambda_{1}$ and $\lambda_{2}$ are the eigenvalues of $A_{H}$. In an open neighborhood $U$ around a nonpseudoumbilical point $p, \lambda_{1}>\lambda_{2}$ on $U$ and $\lambda_{1}, \lambda_{2} \in C^{\infty}(U)$ (in general they are only continuous), and therefore $\mu=\lambda_{1}-\lambda_{2}$ is a positive smooth function on $U$. 
Let $\left\{X_{1}, X_{2}\right\}$ be a local orthonormal frame on $U$ such that $A_{H}\left(X_{1}\right)=\lambda_{1} X_{1}$ and $A_{H}\left(X_{2}\right)=\lambda_{2} X_{2}$. We consider $\omega_{1}^{2}, \omega_{2}^{1} \in \bigwedge^{1}(U)$ defined by

$$
\nabla X_{1}=\omega_{1}^{2} X_{2} \quad \text { and } \quad \nabla X_{2}=\omega_{2}^{1} X_{1} .
$$

Clearly $\omega_{1}^{2}=-\omega_{2}^{1}$. If we put $X=Z=X_{1}$ and $Y=X_{2}$, the Codazzi equation becomes

$$
\begin{aligned}
& R^{N}\left(X_{1}, H, X_{2}, X_{1}\right) \\
& \quad=-\omega_{2}^{1}\left(X_{1}\right) \mu-X_{2} \lambda_{1}-\left\langle B\left(X_{2}, X_{1}\right), \nabla_{X_{1}}^{\perp} H\right\rangle+\left\langle B\left(X_{1}, X_{1}\right), \nabla_{X_{2}}^{\perp} H\right\rangle .
\end{aligned}
$$

Recall that, since $|H|$ is constant, the tangent part of the biharmonic equation is

$$
\operatorname{trace} A_{\nabla ! H}(\cdot)+\operatorname{trace}\left(R^{N}(d \phi(\cdot), H) d \phi(\cdot)\right)^{T}=0 .
$$

Taking the inner product with $X_{2}$, we have

$$
\left\langle B\left(X_{2}, X_{1}\right), \nabla_{X_{1}}^{\perp} H\right\rangle+\left\langle B\left(X_{2}, X_{2}\right), \nabla_{X_{2}}^{\perp} H\right\rangle+R^{N}\left(X_{1}, H, X_{2}, X_{1}\right)=0 ;
$$

thus

$$
\omega_{2}^{1}\left(X_{1}\right) \mu+X_{2} \lambda_{1}=2\left\langle H, \nabla_{X_{2}}^{\perp} H\right\rangle=0
$$

and

$$
\omega_{2}^{1}\left(X_{1}\right)=-\frac{X_{2} \lambda_{1}}{\mu}
$$

Note that

$$
X_{2}\left(\lambda_{2}\right)=X_{2}\left\langle A_{H}\left(X_{2}\right), X_{2}\right\rangle=X_{2}\left\langle B\left(X_{2}, X_{2}\right), H\right\rangle=-X_{2} \lambda_{1},
$$

therefore

$$
\omega_{2}^{1}\left(X_{1}\right)=\frac{1}{2}\left(-\frac{X_{2} \lambda_{1}}{\mu}+\frac{X_{2} \lambda_{2}}{\mu}\right)=-\frac{1}{2} \frac{X_{2} \mu}{\mu} .
$$

Exchanging $X_{1}$ and $X_{2}$, we similarly obtain

$$
\omega_{2}^{1}\left(X_{2}\right)=\frac{1}{2} \frac{X_{1} \mu}{\mu}
$$

therefore

$$
\omega_{2}^{1}=-\frac{1}{2} \frac{X_{2} \mu}{\mu} \omega_{1}+\frac{1}{2} \frac{X_{1} \mu}{\mu} \omega_{2} .
$$

The Gauss equation implies that

$$
d \omega_{2}^{1}\left(X_{1}, X_{2}\right)=K^{M}
$$

that is,

$$
K^{M}=\frac{1}{2}\left(X_{1} X_{1} \ln \mu+X_{2} X_{2} \ln \mu\right)-\left(\omega_{2}^{1}\left(X_{1}\right)\right)^{2}-\left(\omega_{2}^{1}\left(X_{2}\right)\right)^{2},
$$


but

$$
\begin{gathered}
\nabla_{X_{1}} X_{1}=\frac{1}{2}\left(X_{2} \ln \mu\right) X_{2}, \quad\left(\nabla_{X_{1}} X_{1}\right)(\ln \mu)=\frac{1}{2}\left(X_{2} \ln \mu\right)^{2}, \\
\left(\omega_{2}^{1}\left(X_{1}\right)\right)^{2}=\frac{1}{4}\left(X_{2} \ln \mu\right)^{2}=\frac{1}{2}\left(\nabla_{X_{1}} X_{1}\right)(\ln \mu),
\end{gathered}
$$

while

$$
\begin{gathered}
\nabla_{X_{2}} X_{2}=\frac{1}{2}\left(X_{1} \ln \mu\right) X_{1}, \quad\left(\nabla_{X_{2}} X_{2}\right)(\ln \mu)=\frac{1}{2}\left(X_{1} \ln \mu\right)^{2}, \\
\left(\omega_{2}^{1}\left(X_{2}\right)\right)^{2}=\frac{1}{4}\left(X_{1} \ln \mu\right)^{2}=\frac{1}{2}\left(\nabla_{X_{2}} X_{2}\right)(\ln \mu) .
\end{gathered}
$$

Therefore

$$
\Delta \ln \mu=-2 K^{M} .
$$

Since

$$
\left[\frac{1}{\sqrt{\mu}} X_{1}, \frac{1}{\sqrt{\mu}} X_{2}\right]=0
$$

there exist coordinate functions $(x, y)$ on $U$ such that $\partial / \partial x=X_{1} / \sqrt{\mu}$ and $\partial / \partial y=$ $X_{2} / \sqrt{\mu}$. Moreover, the normal part of the biharmonicity equation

$$
\Delta^{\perp} H+\operatorname{trace} B\left(\cdot, A_{H} \cdot\right)+\operatorname{trace}\left(R^{N}(\cdot, H) \cdot\right)^{\perp}=0
$$

implies, when $H$ is constant,

$$
\left|\nabla^{\perp} H\right|^{2}+\left|A_{H}\right|^{2}-\sum_{i=1}^{2} R^{N}\left(X_{i}, H, X_{i}, H\right)=0,
$$

and, since

$$
\lambda_{1}+\lambda_{2}=2|H|^{2} \quad \text { and } \quad \lambda_{1}^{2}+\lambda_{2}^{2}=\left|A_{H}\right|^{2},
$$

we deduce that

$$
\left|A_{H}\right|^{2}-2|H|^{4}=\frac{\left(\lambda_{1}-\lambda_{2}\right)^{2}}{2},
$$

hence

$$
\lambda_{1}-\lambda_{2}=\sqrt{2} \sqrt{\sum_{i=1}^{2} R^{N}\left(X_{i}, H, X_{i}, H\right)-\left|\nabla^{\perp} H\right|^{2}-2|H|^{4}} .
$$

Remark 13. i) If $n=3$, we can replace $\sum_{i=1}^{2} R^{N}\left(X_{i}, H, X_{i}, H\right)$ by $\operatorname{Ricci}^{N}(H, H)$. ii) Let $\phi:\left(M^{2}, g\right) \rightarrow(N, h)$ be a CMC proper-biharmonic Riemannian immersion. If $\left(M^{2}, g\right)$ is complete and has no pseudoumbilical point then its universal cover is (globally) conformally equivalent to $\mathbb{R}^{2}$.

Corollary 14. Let $\phi:\left(M^{2}, g\right) \rightarrow N^{3}(c)$ be a CMC proper-biharmonic Riemannian immersion in a three-dimensional real space form. Then it is umbilical. 
Proof. If there exists a nonumbilical point $p_{0} \in M$, then, around $p_{0}$, we have

$$
\operatorname{Riem}^{N}\left(X_{1}, X_{2}\right)=K^{M}-2|H|^{2}+\frac{1}{2|H|^{2}} \operatorname{Ricci}^{N}(H, H)
$$

and

$$
K^{M}=-\frac{1}{4} \Delta \ln \left(\operatorname{Ricci}^{N}(H, H)-2|H|^{4}\right),
$$

but $\operatorname{Ricci}^{N}(H, H)=2 c|H|^{2}$ is constant, so $K^{M}$ is zero. On the other hand, the first equation implies that $c=K^{M}-2|H|^{2}+c$, which contradicts $K^{M}=0$.

As the formulas for $\lambda_{1}$ and $\lambda_{2}$ in Theorem 12 remain valid also for pseudoumbilical points, we deduce:

Corollary 15. Let $\phi:\left(M^{2}, g\right) \rightarrow\left(N^{3}, h\right)$ be a CMC proper-biharmonic Riemannian immersion. Assume that there exists $c>0$ such that $\operatorname{Ricci}^{N}(U, U) \geq c|U|^{2}$ with $|H|^{2} \in(0, c / 2)$. Then $M^{2}$ has no pseudoumbilical point.

Corollary 16. Let $\phi:\left(M^{2}, g\right) \rightarrow\left(N^{n}, h\right)$ be a CMC proper-biharmonic Riemannian immersion. Assume $M$ is compact, oriented and has no pseudoumbilical point; then $M$ is topologically a torus.

Corollary 17. Let $\phi:\left(M^{2}, g\right) \rightarrow\left(N^{n}, h\right)$ be a proper-biharmonic Riemannian immersion. Assume that $\lambda_{1}$ and $\lambda_{2}$ are constant; then $\nabla A_{H}=0$, and $M$ is flat or pseudoumbilical.

If $M$ is not compact, we need some assumption on the curvature of the target space (see also [Fetcu and Pinheiro 2013, Proposition 4.6 and 4.7]).

Proposition 18. Let $\phi:\left(M^{2}, g\right) \rightarrow\left(N^{n}, h\right)$ be a CMC proper-biharmonic Riemannian immersion. Assume $M$ is noncompact and complete and $K^{M}$ is nonnegative. Assume that $\operatorname{Riem}^{N} \leq K_{0}$, where $K_{0}>0$ (in the sense that $R^{N}(U, V, U, V) \leq K_{0}$ for all $\{U, V\}$ orthonormal). Then $\nabla A_{H}=0$, and $M$ is flat or pseudoumbilical.

Proof. By the previous formulas for the Laplacian of $S_{2}$, we have

$$
\begin{aligned}
-\frac{1}{2} \Delta\left|S_{2}\right|^{2} & =-\left\langle\Delta^{R} S_{2}, S_{2}\right\rangle+\left|\nabla S_{2}\right|^{2} \\
& =K^{M}\left(2\left|S_{2}\right|^{2}-|\tau(\phi)|^{4}\right)+\left|\nabla S_{2}\right|^{2},
\end{aligned}
$$

which must be nonnegative (Remark 7); therefore $\left|S_{2}\right|^{2}$ is a subharmonic function and bounded from above since, for Riemannian immersions,

$$
\left|S_{2}\right|^{2}=8\left(2\left|A_{H}\right|^{2}-3|H|^{4}\right)
$$

and $\left|A_{H}\right|^{2}$ is itself bounded from above. Indeed if $\phi$ is biharmonic, then

$$
\Delta^{\perp} H+\operatorname{trace} B\left(\cdot, A_{H} \cdot\right)+\operatorname{trace}\left(R^{N}(\cdot, H) \cdot\right)^{\perp}=0 ;
$$


thus

$$
\begin{aligned}
\left|A_{H}\right|^{2} & =-\left|\nabla^{\perp} H\right|^{2}+\sum_{i=1}^{2} R^{N}\left(X_{i}, H, X_{i}, H\right) \\
& \leq \sum_{i=1}^{2} R^{N}\left(X_{i}, H, X_{i}, H\right) \leq 2|H|^{2} K_{0},
\end{aligned}
$$

and $\left|A_{H}\right|^{2} \leq 2 K_{0}|H|^{2}$. As $M$ is complete with $K^{M}$ nonnegative, it is parabolic and $\left|S_{2}\right|^{2}$, a subharmonic function bounded from above, must be constant (see [Huber 1957]):

$$
K^{M}\left(\left|A_{H}\right|^{2}-4|H|^{4}\right)=0,
$$

while $\nabla A_{H}=0$; in particular, $\left|A_{H}\right|^{2}$ is constant.

Remark 19. When the dimension of the target is three, we can replace the curvature condition by an upper bound on the Ricci tensor.

The Hopf theorem [1983] shows that a compact simply connected CMC surface immersed in a three-dimensional Euclidean space must be umbilical, hence an embedded round sphere, and the condition of biharmonicity allows us to extend this to any codomain. This result has some strict implications on the set of pseudoumbilical points and hints at the difficulties of working with non-CMC surfaces. An interesting parallel can be drawn with [Fetcu and Pinheiro 2013].

Theorem 20. Let $\phi:\left(M^{2}, g\right) \rightarrow\left(N^{n}, h\right)$ be a proper-biharmonic Riemannian immersion with mean curvature vector field $H$, with $M^{2}$ oriented. Let $z$ be a complex coordinate on $M^{2}$; then the function $\langle B(\partial z, \partial z), H\rangle$ is holomorphic if and only if the norm of $H$ is constant.

Proof. The tangent part of the biharmonic equation is

$$
\operatorname{grad} \frac{|H|^{2}}{2}+\operatorname{trace} A_{\nabla \cdot H}^{\perp}(\cdot)+\operatorname{trace}\left(R^{N}(d \phi(\cdot), H) d \phi(\cdot)\right)^{T}=0 .
$$

Let $g=\lambda^{2}\left(d x^{2}+d y^{2}\right)$ and

$$
\begin{aligned}
\frac{1}{2} \partial x\left(|H|^{2}\right) \partial x+\frac{1}{2} \partial y\left(|H|^{2}\right) \partial y+A_{\nabla \frac{1}{\partial x} H}(\partial x)+A_{\nabla \frac{\perp}{\partial y} H}(\partial y) & \\
& +\left(R^{N}(\partial x, H) \partial x+R^{N}(\partial y, H) \partial y\right)^{T}=0 ;
\end{aligned}
$$

therefore

$$
\frac{\lambda^{2}}{2} \partial x\left(|H|^{2}\right)+\left\langle A_{\nabla \frac{1}{\partial x} H}(\partial x), \partial x\right\rangle+\left\langle A_{\nabla \frac{\perp}{\partial y} H}(\partial y), \partial x\right\rangle+R^{N}(\partial y, H, \partial x, \partial y)=0,
$$

and

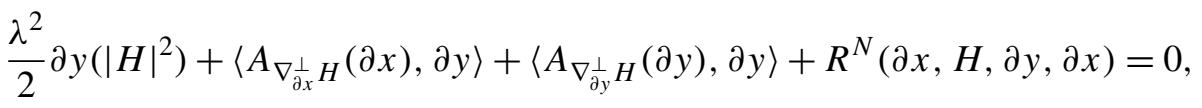


which is equivalent to

(1) $\frac{\lambda^{2}}{2} \partial x\left(|H|^{2}\right)+\left\langle B(\partial x, \partial x), \nabla_{\partial x}^{\perp} H\right\rangle+\left\langle B(\partial x, \partial y), \nabla_{\partial y}^{\perp} H\right\rangle$ $+R^{N}(\partial y, H, \partial x, \partial y)=0$,

and

(2) $\frac{\lambda^{2}}{2} \partial y\left(|H|^{2}\right)+\left\langle B(\partial y, \partial x), \nabla_{\partial x}^{\perp} H\right\rangle+\left\langle B(\partial y, \partial y), \nabla_{\partial y}^{\perp} H\right\rangle$ $+R^{N}(\partial x, H, \partial y, \partial x)=0$.

Since $\partial z=(\partial x-i \partial y) / 2$ and $\partial \bar{z}=(\partial x+i \partial y) / 2$, we see that

$$
B(\partial z, \partial z)=\frac{1}{2}\left(\lambda^{2} H-B(\partial y, \partial y)-i B(\partial x, \partial y)\right)
$$

and

$$
\langle B(\partial z, \partial z), H\rangle=\frac{1}{2}\left(\lambda^{2}|H|^{2}-\langle B(\partial y, \partial y), H\rangle-i\langle B(\partial x, \partial y), H\rangle\right) .
$$

Next we compute $\partial \bar{z}\langle B(\partial z, \partial z), H\rangle$ :

$$
\begin{aligned}
& (\partial x+i \partial y)\left(\lambda^{2}|H|^{2}-\langle B(\partial y, \partial y), H\rangle-i\langle B(\partial x, \partial y), H\rangle\right) \\
& =2 \lambda \frac{\partial \lambda}{\partial x}|H|^{2}+\lambda^{2} \partial x\left(|H|^{2}\right)-\left\langle\nabla_{\partial x}^{\perp} B(\partial y, \partial y), H\right\rangle-\left\langle B(\partial y, \partial y), \nabla_{\partial x}^{\perp} H\right\rangle \\
& +\left\langle\nabla_{\partial y}^{\perp} B(\partial x, \partial y), H\right\rangle+\left\langle B(\partial x, \partial y), \nabla_{\partial y}^{\perp} H\right\rangle \\
& +i\left\{2 \lambda \frac{\partial \lambda}{\partial y}|H|^{2}+\lambda^{2} \partial y\left(|H|^{2}\right)-\left\langle\nabla_{\partial y}^{\perp} B(\partial y, \partial y), H\right\rangle-\left\langle B(\partial y, \partial y), \nabla_{\partial y}^{\perp} H\right\rangle\right. \\
& \left.-\left\langle\nabla_{\partial x}^{\perp} B(\partial x, \partial y), H\right\rangle-\left\langle B(\partial x, \partial y), \nabla_{\partial x}^{\perp} H\right\rangle\right\}
\end{aligned}
$$

$=A+i B$.

With (1),

$$
\begin{aligned}
A=2 \lambda & \frac{\partial \lambda}{\partial x}|H|^{2}+\frac{1}{2} \lambda^{2} \partial x\left(|H|^{2}\right)-\left\langle\nabla_{\partial x}^{\perp} B(\partial y, \partial y), H\right\rangle-\left\langle B(\partial y, \partial y), \nabla_{\partial x}^{\perp} H\right\rangle \\
& +\left\langle\nabla \frac{\perp}{\partial y} B(\partial x, \partial y), H\right\rangle-\left\langle B(\partial x, \partial x), \nabla \frac{\perp}{\partial x} H\right\rangle-R(\partial y, H, \partial x, \partial y) \\
=2 \lambda & \frac{\partial \lambda}{\partial x}|H|^{2}+\frac{1}{2} \lambda^{2} \partial x\left(|H|^{2}\right)-\left\langle\nabla_{\partial x}^{\perp} B(\partial y, \partial y), H\right\rangle+\left\langle\nabla_{\partial y}^{\perp} B(\partial x, \partial y), H\right\rangle \\
& -\left\langle 2 \lambda^{2} H, \nabla_{\partial x}^{\perp} H\right\rangle-R(\partial y, H, \partial x, \partial y) \\
=2 \lambda & \frac{\partial \lambda}{\partial x}|H|^{2}-\frac{1}{2} \lambda^{2} \partial x\left(|H|^{2}\right)-\left\langle\nabla_{\partial x}^{\perp} B(\partial y, \partial y), H\right\rangle+\left\langle\nabla_{\partial y}^{\perp} B(\partial x, \partial y), H\right\rangle \\
& -R(\partial y, H, \partial x, \partial y) .
\end{aligned}
$$


From the Codazzi equation,

$$
\begin{aligned}
\left\langle\nabla_{\partial y}^{\perp} B(\partial x, \partial y), H\right\rangle & \\
= & \left\langle\left(\nabla_{\partial y}^{\perp} B\right)(\partial x, \partial y), H\right\rangle+\left\langle B\left(\nabla_{\partial y} \partial x, \partial y\right), H\right\rangle+\left\langle B\left(\partial x, \nabla_{\partial y} \partial y\right), H\right\rangle \\
= & \left\langle\nabla_{\partial x}^{\perp} B(\partial y, \partial y), H\right\rangle-2\left\langle B\left(\nabla_{\partial x} \partial y, \partial y\right), H\right\rangle+R(\partial y, \partial x, H, \partial y) \\
& \quad+\left\langle B\left(\nabla_{\partial y} \partial x, \partial y\right), H\right\rangle+\left\langle B\left(\partial x, \nabla_{\partial y} \partial y\right), H\right\rangle
\end{aligned}
$$

therefore

$$
\begin{aligned}
A=2 \lambda & \frac{\partial \lambda}{\partial x}|H|^{2}-\frac{1}{2} \lambda^{2} \partial x\left(|H|^{2}\right)-\left\langle\nabla_{\partial x}^{\perp} B(\partial y, \partial y), H\right\rangle+\left\langle\nabla_{\partial x}^{\perp} B(\partial y, \partial y), H\right\rangle \\
& -\left\langle B\left(\nabla_{\partial x} \partial y, \partial y\right), H\right\rangle+\left\langle B\left(\partial x, \nabla_{\partial y} \partial y\right), H\right\rangle+R(\partial y, \partial x, H, \partial y) \\
& -R(\partial y, H, \partial x, \partial y) \\
=2 \lambda & \frac{\partial \lambda}{\partial x}|H|^{2}-\frac{1}{2} \lambda^{2} \partial x\left(|H|^{2}\right)-\left\langle B\left(\frac{1}{\lambda}\left(\frac{\partial \lambda}{\partial y} \partial x+\frac{\partial \lambda}{\partial x} \partial y\right), \partial y\right), H\right\rangle \\
& +\left\langle B\left(\frac{1}{\lambda}\left(-\frac{\partial \lambda}{\partial x} \partial x+\frac{\partial \lambda}{\partial y} \partial y\right), \partial x\right), H\right\rangle \\
=- & \frac{1}{2} \lambda^{2} \partial x\left(|H|^{2}\right) .
\end{aligned}
$$

Identical arguments for the imaginary part $B$, using (2), yield

$$
B=\frac{1}{2} \lambda^{2} \partial y\left(|H|^{2}\right) .
$$

Remark 21. If $\phi:\left(M^{2}, g\right) \rightarrow\left(N^{n}, h\right)$ is a CMC proper-biharmonic Riemannian immersion, with $M^{2}$ oriented. Then $\langle B(\partial z, \partial z), H\rangle d z^{2}$ is globally defined and, if $M^{2}$ has no pseudoumbilical point, it is equal to $d z^{2} / 4$ and therefore $M^{2}$ is an affine manifold.

Corollary 22. Let $\phi:\left(M^{2}, g\right) \rightarrow\left(N^{n}, h\right)$ be a CMC proper-biharmonic Riemannian immersion, with $M^{2}$ oriented. If $M^{2}$ is not pseudoumbilical, then its pseudoumbilical points are isolated.

Theorem 20 yields:

Theorem 23. Let $\phi:\left(M^{2}, g\right) \rightarrow\left(N^{n}, h\right)$ be a CMC proper-biharmonic Riemannian immersion. If $M^{2}$ is a topological sphere $\mathbb{S}^{2}$, then $M$ is pseudoumbilical.

Proof. Since $\langle B(\partial z, \partial z), H\rangle=0$, we have

$$
\langle B(\partial x, \partial x)-B(\partial y, \partial y), H\rangle=0 \quad \text { and } \quad\langle B(\partial x, \partial y), H\rangle=0,
$$

which is equivalent to

$$
\left\langle A_{H}(\partial x), \partial x\right\rangle=\left\langle A_{H}(\partial y), \partial y\right\rangle \quad \text { and } \quad\left\langle A_{H}(\partial x), \partial y\right\rangle=\left\langle A_{H}(\partial y), \partial x\right\rangle=0 .
$$




\section{References}

[Baird et al. 2011] P. Baird, E. Loubeau, and C. Oniciuc, "Harmonic and biharmonic maps from surfaces", pp. 223-230 in Harmonic maps and differential geometry (Cagliari, 2009), edited by E. Loubeau and S. Montaldo, Contemp. Math. 542, Amer. Math. Soc., Providence, RI, 2011. MR 2012e:53121 Zbl 1236.58024

[Fetcu and Pinheiro 2013] D. Fetcu and A. L. Pinheiro, "Biharmonic surfaces with parallel mean curvature in complex space forms", preprint, 2013. arXiv 1303.4279v1

[Hasanis and Vlachos 1996] T. Hasanis and T. Vlachos, "2-type surfaces in a hypersphere", Kodai Math. J. 19:1 (1996), 26-38. MR 96m:53059 Zbl 0856.53019

[Hopf 1983] H. Hopf, Differential geometry in the large, Lecture Notes in Math. 1000, Springer, Berlin, 1983. MR 85b:53001 Zbl 0526.53002

[Huber 1957] A. Huber, "On subharmonic functions and differential geometry in the large", Comment. Math. Helv. 32 (1957), 13-72. MR 20 \#970 Zbl 0080.15001

[Jiang 1986] G. Y. Jiang, "2-harmonic maps and their first and second variational formulas", Chinese Ann. Math. (A) 7:4 (1986), 389-402. In Chinese; summary translated in Chinese Ann. Math. (B) 7:4 (1986), p. 523. MR 88i:58039 Zbl 0628.58008

[Jiang 1987] G. Y. Jiang, "The conservation law for 2-harmonic maps between Riemannian manifolds", Acta Math. Sinica 30:2 (1987), 220-225. In Chinese. MR 88k:58028 Zbl 0631.58007

[Loubeau et al. 2008] E. Loubeau, S. Montaldo, and C. Oniciuc, "The stress-energy tensor for biharmonic maps", Math. Z. 259:3 (2008), 503-524. MR 2009c:58020 Zbl 1139.58010

[Montaldo and Oniciuc 2006] S. Montaldo and C. Oniciuc, "A short survey on biharmonic maps between Riemannian manifolds", Rev. Un. Mat. Argentina 47:2 (2006), 1-22. MR 2008a:53063 Zbl 1140.58004

[Ou and Wang 2011] Y.-L. Ou and Z.-P. Wang, "Constant mean curvature and totally umbilical biharmonic surfaces in 3-dimensional geometries", J. Geom. Phys. 61:10 (2011), 1845-1853. MR 2012g:53120 Zbl 1227.58004

[Sasahara 2007] T. Sasahara, "Biharmonic Lagrangian surfaces of constant mean curvature in complex space forms”, Glasg. Math. J. 49:3 (2007), 497-507. MR 2008i:53081 Zbl 1132.53310

Received June 11, 2013.

ERIC LOUBEAU

DÉPARTEMENT DE MATHÉMATIQUES

UNIVERSITÉ DE BRETAGNE OCCIDENTALE

6, AVEnue Victor Le Gorgeu

CS 93837

29238 BREST 3

FRANCE

loubeau@univ-brest.fr

Cezar Oniciuc

FACULTY OF MATHEMATICS

ALEXANDRU IOAN CUZA UNIVERSITY OF IASI

Boulevard Carol I, Number 11

700506 IASI

ROMANIA

oniciucc@uaic.ro 


\title{
PACIFIC JOURNAL OF MATHEMATICS
}

\author{
msp.org/pjm
}

Founded in 1951 by E. F. Beckenbach (1906-1982) and F. Wolf (1904-1989)

\section{EDITORS}

Don Blasius (Managing Editor)

Department of Mathematics

University of California

Los Angeles, CA 90095-1555

blasius@math.ucla.edu

\author{
Paul Balmer \\ Department of Mathematics \\ University of California \\ Los Angeles, CA 90095-1555 \\ balmer@math.ucla.edu \\ Robert Finn \\ Department of Mathematics \\ Stanford University \\ Stanford, CA 94305-2125 \\ finn@math.stanford.edu \\ Sorin Popa \\ Department of Mathematics \\ University of California \\ Los Angeles, CA 90095-1555 \\ popa@math.ucla.edu
}

\author{
Vyjayanthi Chari \\ Department of Mathematics \\ University of California \\ Riverside, CA 92521-0135 \\ chari@math.ucr.edu \\ Kefeng Liu \\ Department of Mathematics \\ University of California \\ Los Angeles, CA 90095-1555 \\ liu@math.ucla.edu \\ Jie Qing \\ Department of Mathematics \\ University of California \\ Santa Cruz, CA 95064 \\ qing@ cats.ucsc.edu
}

\section{PRODUCTION}

Silvio Levy, Scientific Editor, production@msp.org

\section{SUPPORTING INSTITUTIONS}

ACADEMIA SINICA, TAIPEI

CALIFORNIA INST. OF TECHNOLOGY

INST. DE MATEMÁTICA PURA E APLICADA

KEIO UNIVERSITY

MATH. SCIENCES RESEARCH INSTITUTE

NEW MEXICO STATE UNIV.

OREGON STATE UNIV.

\author{
STANFORD UNIVERSITY \\ UNIV. OF BRITISH COLUMBIA \\ UNIV. OF CALIFORNIA, BERKELEY \\ UNIV. OF CALIFORNIA, DAVIS \\ UNIV. OF CALIFORNIA, LOS ANGELES \\ UNIV. OF CALIFORNIA, RIVERSIDE \\ UNIV. OF CALIFORNIA, SAN DIEGO \\ UNIV. OF CALIF., SANTA BARBARA
}

\author{
Daryl Cooper \\ Department of Mathematics \\ University of California \\ Santa Barbara, CA 93106-3080 \\ cooper@math.ucsb.edu \\ Jiang-Hua Lu \\ Department of Mathematics \\ The University of Hong Kong \\ Pokfulam Rd., Hong Kong \\ jhlu@maths.hku.hk \\ Paul Yang \\ Department of Mathematics \\ Princeton University \\ Princeton NJ 08544-1000 \\ yang@math.princeton.edu
}

These supporting institutions contribute to the cost of publication of this Journal, but they are not owners or publishers and have no responsibility for its contents or policies.

See inside back cover or msp.org/pjm for submission instructions.

The subscription price for 2014 is US $\$ 410 /$ year for the electronic version, and \$535/year for print and electronic.

Subscriptions, requests for back issues and changes of subscribers address should be sent to Pacific Journal of Mathematics, P.O. Box 4163, Berkeley, CA 94704-0163, U.S.A. The Pacific Journal of Mathematics is indexed by Mathematical Reviews, Zentralblatt MATH, PASCAL CNRS Index, Referativnyi Zhurnal, Current Mathematical Publications and Web of Knowledge (Science Citation Index).

The Pacific Journal of Mathematics (ISSN 0030-8730) at the University of California, c/o Department of Mathematics, 798 Evans Hall \#3840, Berkeley, CA 94720-3840, is published twelve times a year. Periodical rate postage paid at Berkeley, CA 94704, and additional mailing offices. POSTMASTER: send address changes to Pacific Journal of Mathematics, P.O. Box 4163, Berkeley, CA 94704-0163.

PJM peer review and production are managed by EditFLOW ${ }^{\circledR}$ from Mathematical Sciences Publishers.

\section{PUBLISHED BY}

\section{mathematical sciences publishers \\ nonprofit scientific publishing}

http://msp.org/

(C) 2014 Mathematical Sciences Publishers 


\section{PACIFIC JOURNAL OF MATHEMATICS}

Volume $271 \quad$ No. $1 \quad$ September 2014

Proper holomorphic maps between bounded symmetric domains revisited 1

GAUTAM BHARALI and JAIKRISHNAN JANARDHANAN

An explicit Majorana representation of the group $3^{2}: 2$ of $3 C$-pure type 25

HSIAN-YANG CHEN and CHING HUNG LAM

Sofic groups: graph products and graphs of groups

LAUra Ciobanu, DereK F. Holt and SARAh ReES

Perturbations of a critical fractional equation

Eduardo Colorado, Arturo de Pablo and Urko SÁnchez

A density theorem in parametrized differential Galois theory

THOMAS DREYFUS

On the classification of complete area-stationary and stable surfaces in $\quad 143$ the subriemannian Sol manifold

MATteo Galli

Periodic orbits of Hamiltonian systems linear and hyperbolic at infinity

BAŞAK Z. GÜREL

Nonsplittability of the rational homology cobordism group of

3-manifolds

SE-Goo KIM and CHARLES LIVINGSTON

Biharmonic surfaces of constant mean curvature

ERIC LOUBEAU and CEZAR ONICIUC

Foliations of a smooth metric measure space by hypersurfaces with constant $f$-mean curvature

JUNCHEOL PYO

On the existence of large degree Galois representations for fields of small 243 discriminant

JEREMY ROUSE and FRANK THORNE 